\title{
Local Strain Distribution Arising in Shape Memory Alloy Composite Subjected to Thermal Loading
}

\author{
Go Murasawa $^{1, *}$ and Satoru Yoneyama ${ }^{2}$ \\ ${ }^{1}$ Department of Mechanical Engineering, Yamagata University, Yonezawa 992-8510, Japan \\ ${ }^{2}$ Department of Mechanical Systems Engineering, Osaka Prefecture University, Sakai 599-8531, Japan
}

\begin{abstract}
The main purpose of the present research is the measurement of local strain distributions generated in a shape memory alloy composite (SMAC) under thermal loading. In the present study, firstly, the test system is constructed on the basis of Digital Image Correlation (D.I.C.) in order to measure local strains all over the surface of object. Then, local strain distributions generated in SMAC are measured under heating. Results in present study are as follows: (1) Longitudinal local strain reveals inhomogeneous deformation behavior during austenite transformation of SMA fiber, and shows distribution along to $x$ and $y$ direction after austenite transformation. Also, we can see that the state of distribution is different between $x$ and $y$ directions after austenite transformation. (2) Lateral local strain begins to generate around fiber during austenite transformation of SMA, and shows distribution along to only $y$ direction after austenite transformation. This distribution is caused by thermal expansion of matrix. (3) Shearing local strain begins to generate at fiber edge during austenite transformation of SMA fiber, and shows distribution along to $x$ and $y$ direction after austenite transformation.
\end{abstract}

(Received August 29, 2005; Accepted January 31, 2006; Published March 15, 2006)

Keywords: NiTi, epoxy, composite, internal stress, deformation, local strain, measurement, distribution, digital image correlation

\section{Introduction}

Many researchers have been conducting investigations about basic performances and applications of shape memory alloy (SMA). Therefore, there have been many results of research about SMA. ${ }^{1-7)}$ In the recent years, some researchers have studied about shape memory alloy composite (SMAC) which consists of SMA reinforcement and polymer or metal matrix. ${ }^{8-22)}$ Especially, considerable attention has been paid to the creating of internal stress in matrix under thermal loading. ${ }^{8-11)}$ On the other hand, SMAC exhibits deformation of composite under thermal loading. ${ }^{14,19,20)}$ In the case of controlling both of internal stress and deformation, it is important to study about their thermo-mechanical behavior from the viewpoint of analysis and experiment. Umezaki et $a{ }^{21)}$ tried to measure the distribution of deformation on the surface of SMAC during thermal loading. Ro et al. ${ }^{22)}$ analytically investigated temperature distribution arising in SMAC during thermal loading. Also, present authors analytically investigated the change of internal stress and deformation in SMAC during thermal loading, and experimentally tried to measure their values. ${ }^{19,20)}$ As a result, it was seen that internal stress and deformation had distribution in SMAC, and we could not predict their value sufficiently. Therefore, it is necessary to make the distribution of internal stress and deformation in SMAC clear. If we can measure the internal stress and deformation in SMAC, and construct new analytical model considering distribution of them, we can approach the "control of internal stress and deformation in structure".

The main purpose of the present research is the measurement of local strain distributions generated in SMAC under thermal loading. In the present study, firstly, the test system is constructed on the basis of Digital Image Correlation (D.I.C.) in order to measure local strains all over the surface of object.

*Corresponding author, E-mail: murasawa@yz.yamagata-u.ac.jp
D.I.C. is one of the measurement methods, which can measure the distribution of displacement all over the subject. $^{23,24)}$ We can measure distributions of local strain such as longitudinal, lateral and shearing local strain generated on material by using this system. Secondary, local strain distributions generated in SMAC are measured under heating. Results in present study are as follows: (1) Longitudinal local strain reveals inhomogeneous deformation behavior during austenite transformation of SMA fiber, and shows distribution along to $x$ and $y$ direction after austenite transformation. Also, we can see that the state of distribution is different between $x$ and $y$ directions after austenite transformation. (2) Lateral local strain begins to generate around fiber during austenite transformation of SMA, and shows distribution along to only $y$ direction after austenite transformation. This distribution is caused by thermal expansion of matrix. (3) Shearing local strain begins to generate at fiber edge during austenite transformation of SMA fiber, and shows distribution along to $x$ and $y$ direction after austenite transformation.

\section{Fabrication of Shape Memory Alloy Composite}

NiTi alloy and epoxy resin are adopted as SMA fiber and matrix material in SMAC. NiTi fiber $($ diameter $=1.0 \mathrm{~mm})$ shows shape memory effect (SME) at room temperature, and its austenite finish temperature is about $90^{\circ} \mathrm{C}$. Then, SME single fiber/Epoxy matrix composites are fabricated by casting epoxy resin (Epoxy:Hardner = 93:7) into a mold fixed pre-strained SMA fiber. The glass transition temperature of the epoxy resin is $70^{\circ} \mathrm{C}$. An amount of pre-strain given to SMA fiber is determined from thermo-mechanical loading tests such as recovery stress test and recovery strain test of SMA. ${ }^{19,20)}$ Adequate pre-strain of SMA fiber in composite is 0.05 in present study. Figure 1 shows specimen configuration of composite. 

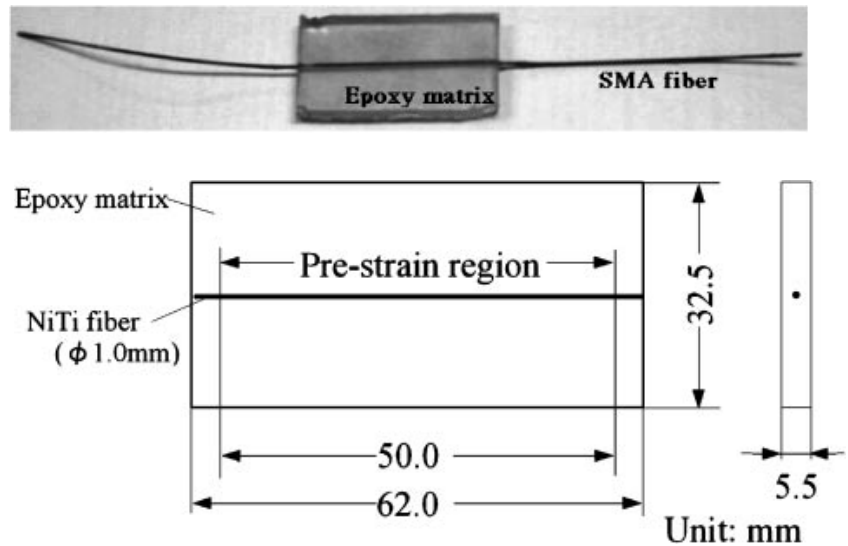

Fig. 1 Specimen configuration.

\section{Methods}

\subsection{Joule heating}

Joule heating is heating method by using electric current. Direct current stabilization power source (KENWOOD Ltd.) is used to heat SMA fiber. The temperature during joule heating is determined from the following Madill's equation. ${ }^{25)}$

$$
\rho c V \frac{d T}{d t}=R i^{2}(t)-h A\left(T-T_{a}\right)
$$

where, $\rho=$ density of wire material $\left[\mathrm{kgm}^{-3}\right], c=$ specific heat of wire $\left[\mathrm{Jkg}^{-1}{ }^{\circ} \mathrm{C}^{-1}\right], V=$ volume of wire $\left[\mathrm{m}^{3}\right], T=$ wire temperature $\left[{ }^{\circ} \mathrm{C}\right], t=$ time $[\mathrm{s}], I=$ electric current $[\mathrm{A}]$, $R=$ electric resistance $[\Omega], \mathrm{h}=$ convection heat transfer coefficient $\left[\mathrm{Wm}^{-2}{ }^{\circ} \mathrm{C}^{-1}\right], A=$ surface, area of wire $\left[\mathrm{m}^{2}\right]$, $T_{a}=$ room temperature $\left[{ }^{\circ} \mathrm{C}\right]$. Firstly, the temperature of SMA fiber is investigated during joule heating to determine a temperature according to electric current value. Figure 2(a) shows temperature-time relation at several electric current values during joule heating. Temperature of SMA fiber is measured by using infrared radiation thermometry method. As shown in Fig. 2(a), temperature reaches equilibrium state after $5 \mathrm{~min}$ for almost of electric current values. Therefore, electricity is gradually increased every $5 \mathrm{~min}$ up to $95^{\circ} \mathrm{C}$ as shown in Fig. 2(a) when we heat SMA fiber in composite.

Also, $d T / d t$ is 0 and $i(t)$ is $i$ in eq. (1) in this heating method. So, we can obtain the following linear relationship between $T-T_{a}$ and $i^{2}$.

$$
T-T_{a}-\alpha i^{2}, \alpha=R / h A
$$

Figure 2(b) shows relation between the difference of temperatures and square of electric current value at equilibrium state for SMA fiber under joule heating. As shown in this figure, results show linear relationship between $T-T_{a}$ and $i^{2}$, and its slope is 5.16. We can estimate a temperature according to electric current value during joule heating.

\subsection{Measurement of local strain distribution}

Test system is constructed on the basis of D.I.C. ${ }^{23,24)}$ in order to measure local strains. This system consists of three parts as follows: (A) Image acquisition, (B) Digital image correlation (calculation of displacement distribution), (C)

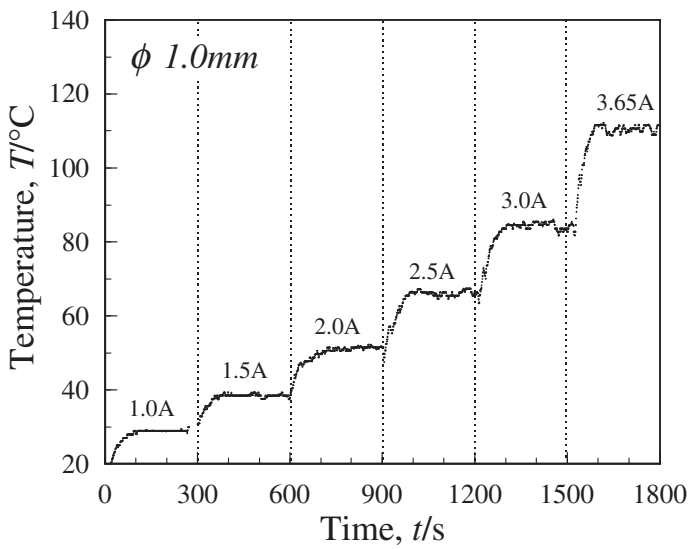

(a)

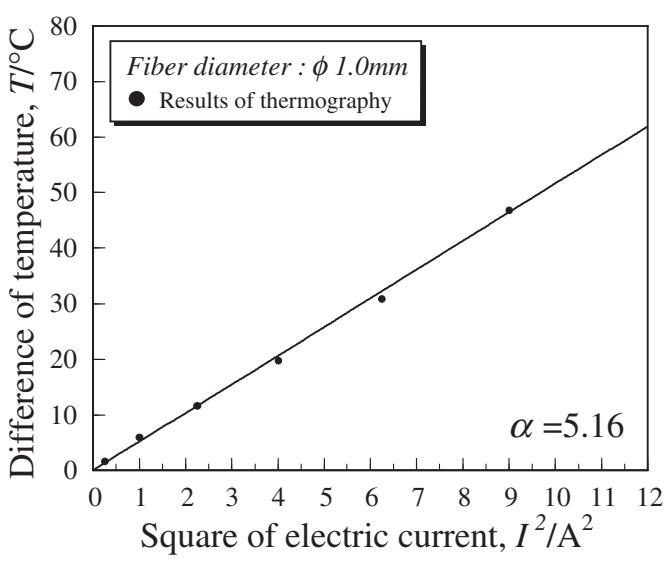

(b)

Fig. 2 (a) Temperature-time relation during joule heating. (b) Difference of temperature-square of electric current value relation during joule heating.

Calculation of local strain distribution. Figure 3 shows a flow chart up to calculating local strain distribution. Also, apparatus used in this system are indicated in this figure. Firstly, images used in D.I.C. are taken at an interval during deformation of specimen (A). Secondary, two images are selected from those images, and displacement all over the surface of specimen can be calculated by comparing these two images (i.e., undeformed image and deformed image) in D.I.C. (B). Thirdly, local strain all over the surface of specimen is calculated from the displacement all over the surface of specimen (C). Calculation method of local strain is following as shown in Fig. 4.

\subsubsection{Approximation of displacement distribution}

Region of displacement distribution [Fig. 4(a)] is divided into several small regions $(1,2,3, \ldots)$ like Fig. 4(b). Every small region (displacement distribution area) is approximated with 2-order curved surface. The center area in approximated displacement distribution area without surroundings of boundary is extracted, and its each area is combined, respectively.

\subsubsection{Local strain distributions}

Local strain distribution is mathematically calculated from approximate curved surface as using following equations, 


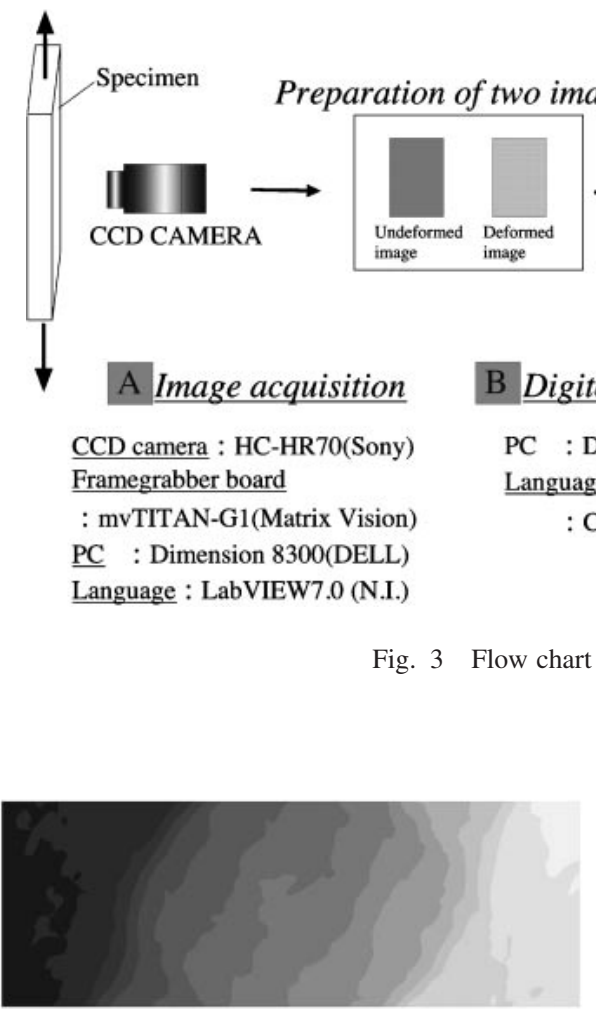

(a)Displacement distribution obtained from D.I.C.

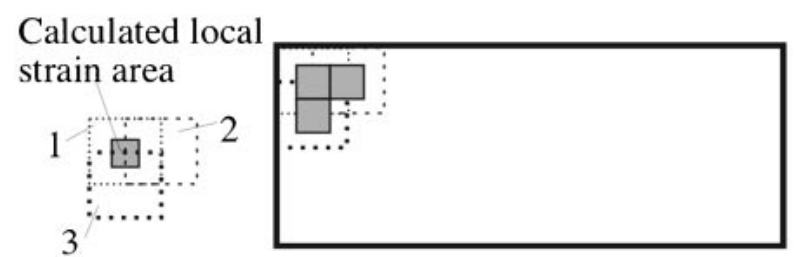

(b)Calculation method of approximated displacement distribution and local strain distribution

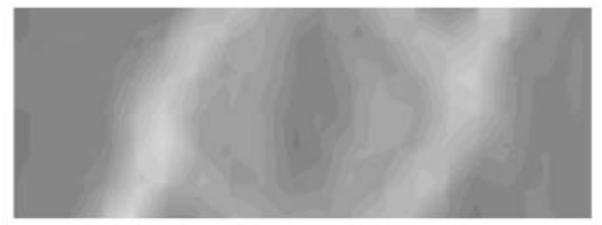

(c)Local strain distribution

Fig. 4 Method of calculating local strain distribution.

$$
\varepsilon_{x}=\frac{\partial u_{x}}{\partial x}, \varepsilon_{y}=\frac{\partial u_{y}}{\partial y}, \varepsilon_{x y}=\frac{1}{2}\left(\frac{\partial u_{x}}{\partial y}+\frac{\partial u_{y}}{\partial x}\right)
$$

Figure 4(c) shows local strain distribution obtained from above calculating method.

\subsection{Experiment}

Figure 5(a) shows experimental setup. Pre-strained SME single fiber/Epoxy matrix composite is gradually heated up to about $95^{\circ} \mathrm{C}$. Only fiber in composite is heated by joule heating method. Time to turn on electricity is $5 \mathrm{~min}$ at an interval. 17 pictures are taken at several temperatures up to about $95^{\circ} \mathrm{C}$ by using CCD camera. Subset size is $31 \times 31$ pixels and searching area size is $39 \times 39$ pixels in D.I.C. These fittest sizes are determined from following test. The specimen is moved in parallel by the tensile machine, and the displacement obtained from D.I.C. is compared with that obtained from the crosshead of tensile machine for several subset sizes and searching area sizes.

\section{Results}

SMAC is heated up to about $95^{\circ} \mathrm{C}$, and local strain distributions are measured at an area on specimen as shown in Fig. 5(b). Figure 6 shows schematic illustration of macroscopic deformation-temperature relation of SMAC under heating. The temperature in Fig. 6 is that of SMA fiber in composite. SMAC creates internal stress in matrix, and simultaneously shows macroscopic deformation during heating. Macroscopic deformations of SMAC are thermal expansion up to $65^{\circ} \mathrm{C}$, shrinkage due to austenite transformation of SMA fiber from 65 to $90^{\circ} \mathrm{C}$ and thermal expansion again after $90^{\circ} \mathrm{C}$ during heating. Figure 7 shows local strain distributions arising in SMAC under heating. Results of local strain distribution are shown at temperature regions of thermal expansion, austenite transformation and after austenite transformation of SMA fiber in composite. Also, results of longitudinal local strain, lateral local strain and shearing local strain are given in Figs. 7(a), (b) and (c). As shown in these figures, the distribution of all strains shows homogeneous deformation behavior at thermal expansion region. Next, from even a cursory examination of figures at austenite transformation region, longitudinal local strain reveals one-sided distribution. On the other hand, lateral local strain begins to generate around SMA fiber, and shearing local strain begins to generate at fiber edge. Finally, at region 


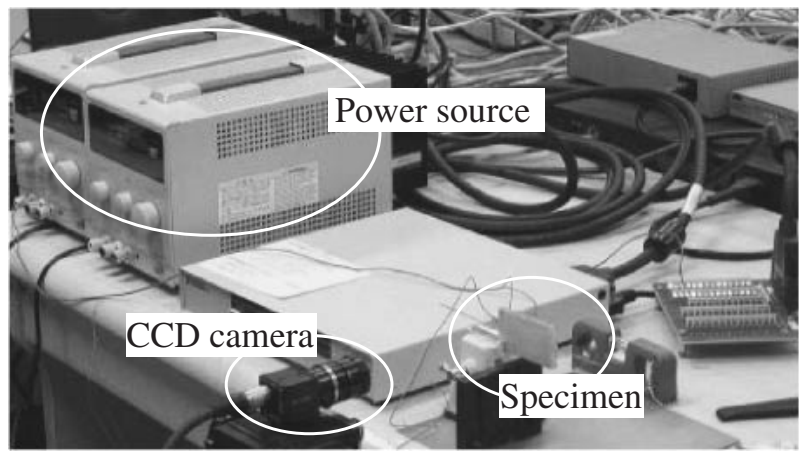

(a) Experimental setup

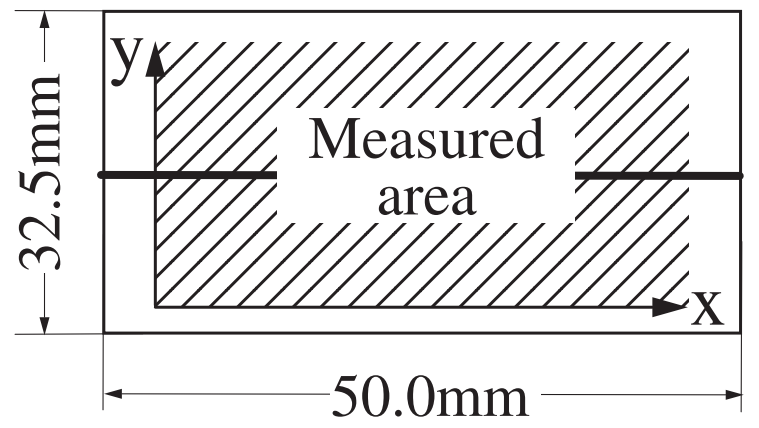

(b) Measured local strain area

Fig. 5 (a) Experimental setup. (b) Measured local strain area.

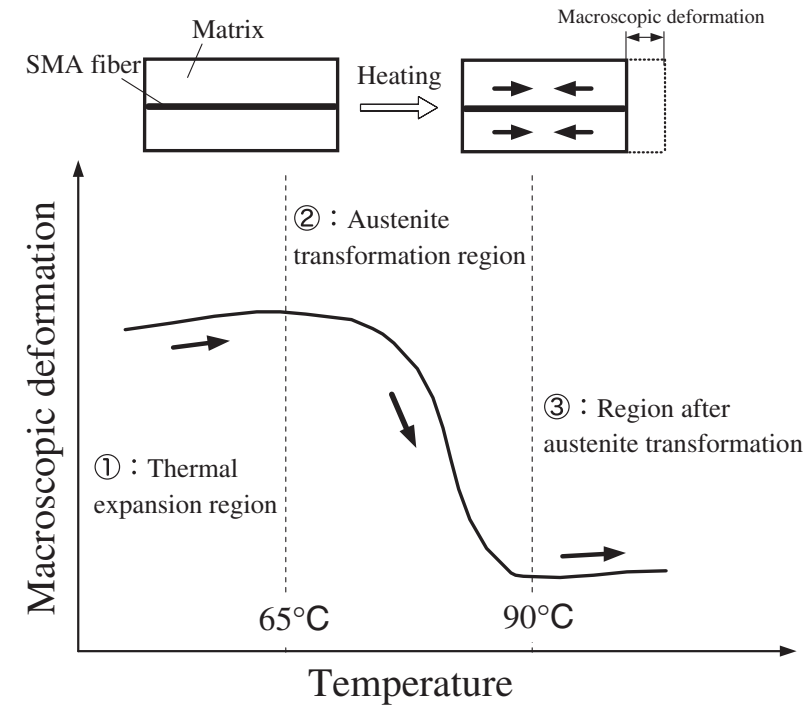

Fig. 6 Schematic illustration of macroscopic deformation-temperature relation of SMAC under heating.

after austenite transformation, longitudinal local strain and shearing local strain show distribution along to $x$ and $y$ direction. Lateral local strain shows distribution along to only $y$ direction.

\section{Discussion}

Local strains along to $x$ or $y$ direction are shown in Fig. 8 . As shown in these figures, we can also find that longitudinal
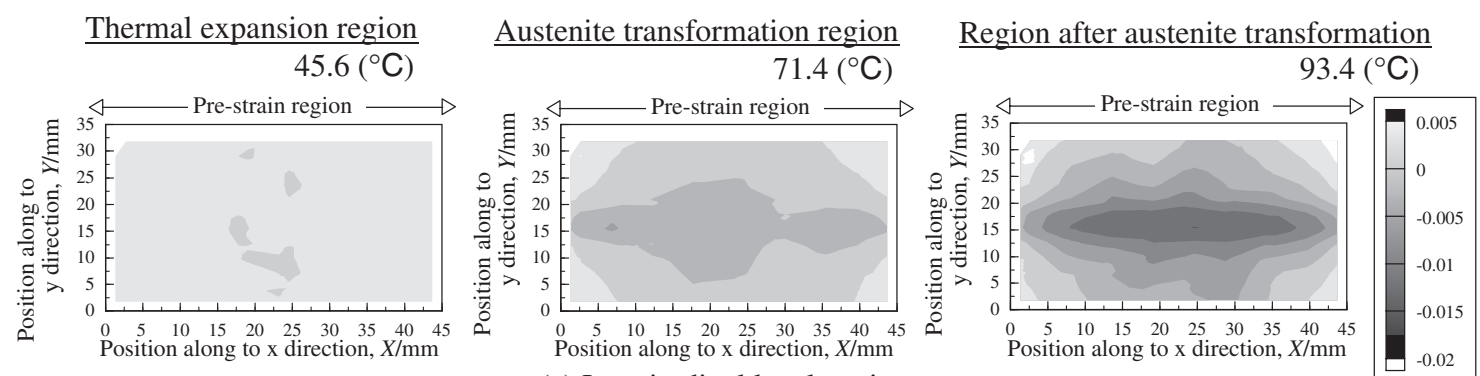

(a) Longitudinal local strain
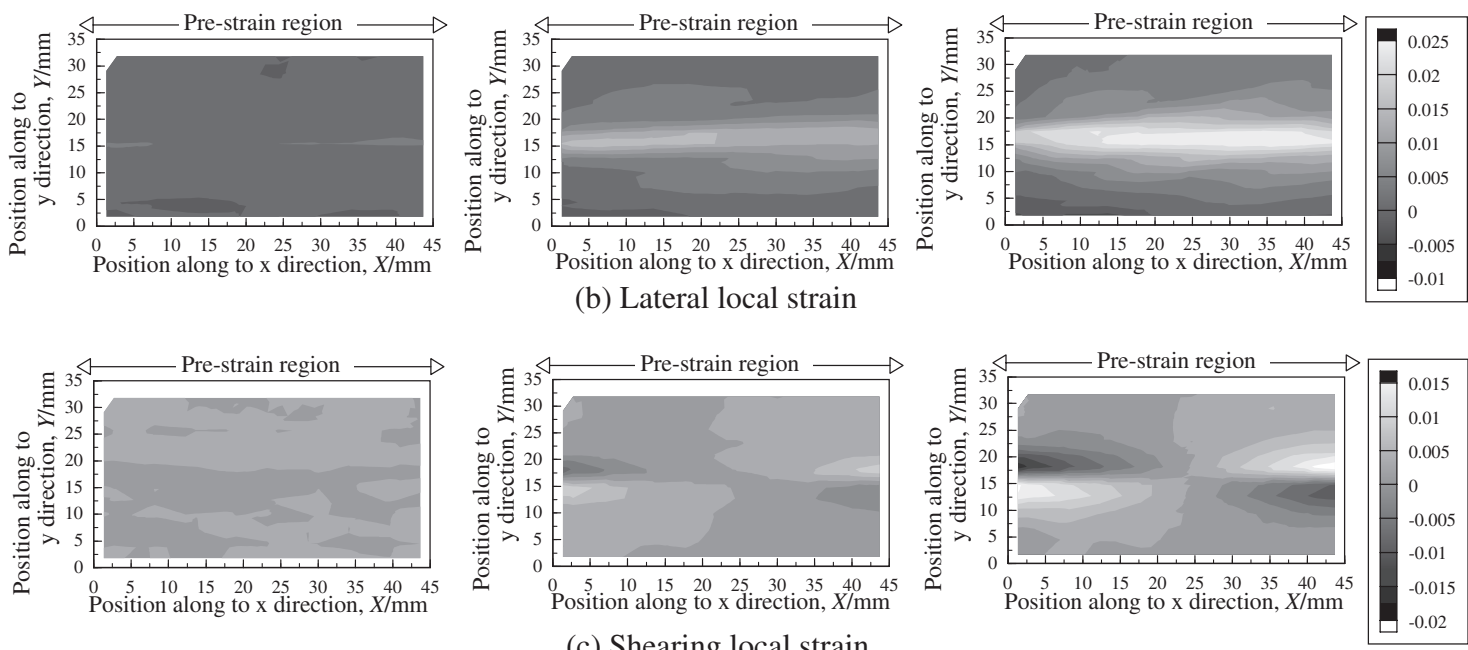

(c) Shearing local strain

Fig. 7 Local strain distribution on the surface of SMAC during thermo-loading. 

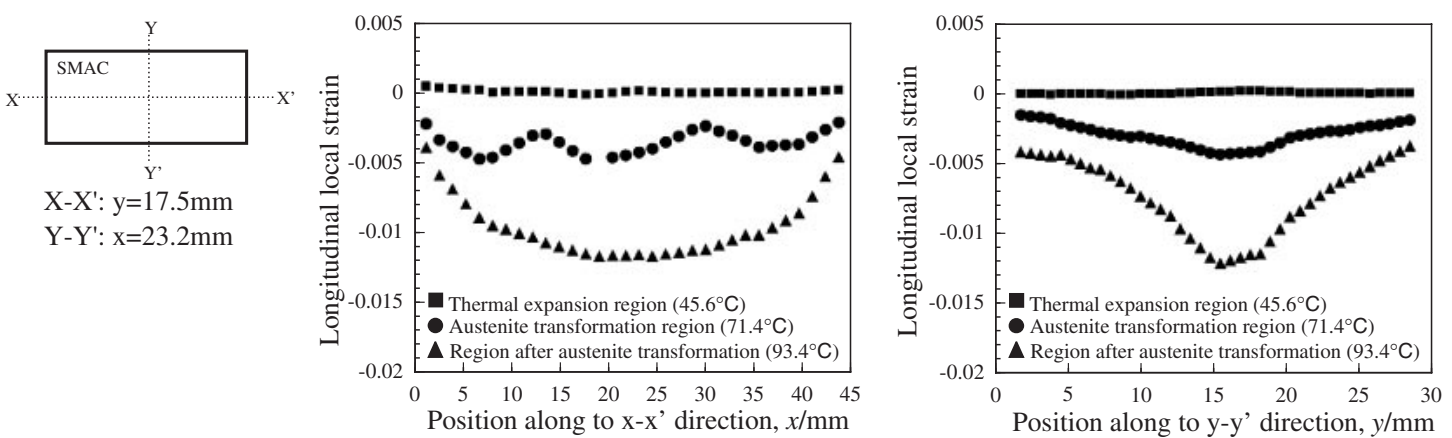

(a) Longitudinal local strain

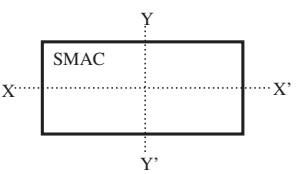

$X-X^{\prime}: y=17.5 \mathrm{~mm}$ $Y-Y^{\prime}: x=23.2 \mathrm{~mm}$
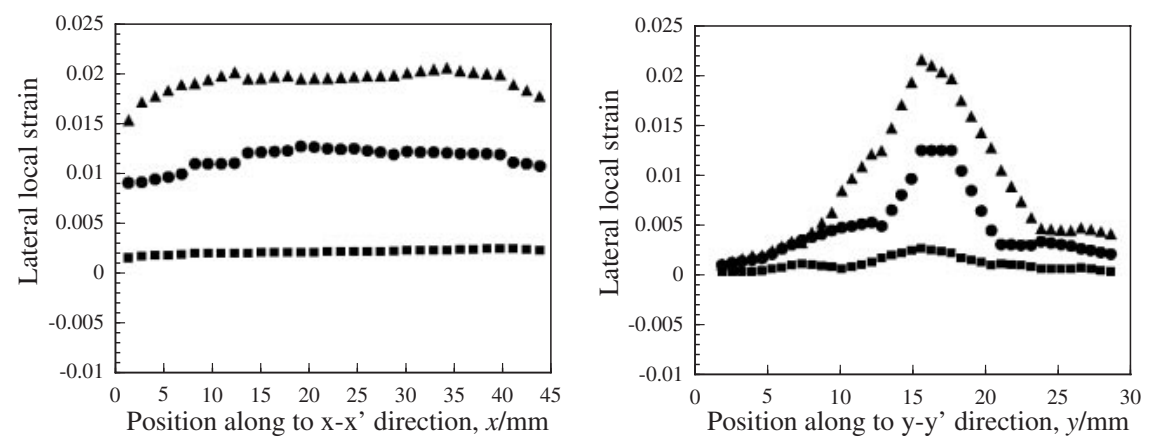

(b) Lateral local strain
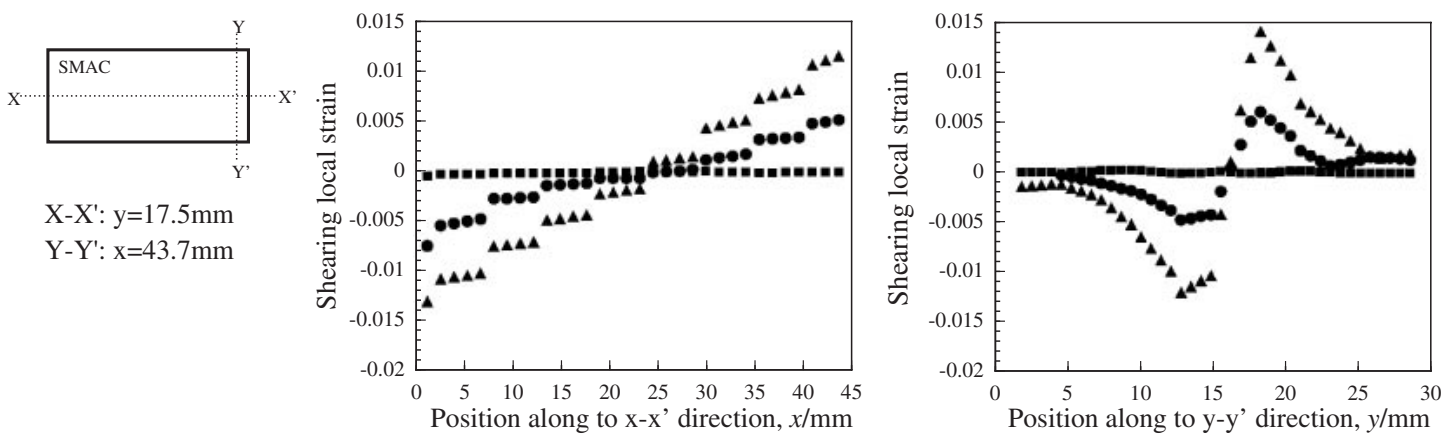

(c) Shearing local strain

Fig. 8 Local strains along to $x$ or $y$ direction on the surface of SMAC during heating.

and shearing local strains show distribution along to $x$ and $y$ direction, and lateral local strain shows distribution along to only $y$ direction.

Longitudinal local strain reveals inhomogeneous deformation behavior during austenite transformation of SMA fiber as shown in Fig. 8(a). This inhomogeneous deformation behavior is derived from that of SMA fiber itself such as nucleation and propagation of phase transformation. ${ }^{5-7)}$ Also, we can see that the state of distribution is different between $x$ and $y$ directions. When we construct an analytical model considering the distribution of deformation in SMAC, it is difficult to consider inhomogeneous deformation during austenite transformation of fiber. On the other hand, the local strain shows simple distribution after austenite transformation of fiber. Then, it is possible to construct an analytical model only in the case of "after austenite transformation".

Lateral local strain begins to generate around fiber during austenite transformation of SMA, and shows distribution only along to $y$ direction after austenite transformation as shown in Fig. 8(b). Then, the distribution along to $y$ direction is almost linear. Lateral local strain distribution is caused by thermal expansion of matrix.

\section{Conclusion}

Local strain distributions generated in Pre-strained SME single fiber/Epoxy matrix composite are measured under thermal loading. The results are as follows:

(1) Longitudinal local strain reveals inhomogeneous deformation behavior during austenite transformation of SMA fiber, and shows distribution along to $x$ and $y$ direction after austenite transformation. Also, we can see that the state of distribution is different between $x$ and $y$ directions.

(2) Lateral local strain begins to generate around fiber during austenite transformation of SMA, and shows distribution along to only $y$ direction after austenite transformation. This distribution is caused by thermal expansion of matrix.

(3) Shearing local strain begins to generate at fiber edge during austenite transformation of SMA fiber, and 
shows distribution along to $x$ and $y$ direction after austenite transformation.

\section{Acknowledgements}

The author would like to express my deep gratitude to Prof. Keiichiro Tohgo (Shizuoka University, Japan) and Prof. Masahisa Takashi (Aoyama Gakuin University, Japan) for a lot of supports.

\section{REFERENCES}

1) K. Tanaka: Res. Mechanica 18 (1986) 251-263.

2) D. S. Ford and S. R. White: Acta Mater. 44 (1993) 2295-2307.

3) L. C. Binson: J. Intell. Mater. Syst. and Struct. 4 (1993) 229-242.

4) H. Inoue, N. Miwa and N. Inakazu: Acta Mater. 44 (1996) 4825-4834.

5) J. A. Shaw and S. Kyriakides: Acta Mater. 45 (1997) 683-700.

6) G. Murasawa, M. Koushinbou, S. Yoneyama, T. Sakuma and M. Takashi: Proc. 2004 SEM X International Congress (2004) CDROM publication.

7) G. Murasawa, S. Yoneyama, T. Sakuma and M. Takashi: Proc. 2005 SEM Annual Conference (2005) CD-ROM publication.

8) Y. Furuya, A. Sasaki and M. Taya: Mater. Trans., JIM 34 (1993) 224227.

9) A. Shimamoto and Y. Furuya: Proc. ICCM-11 6 (1997) 493-499.

10) W. D. Armstrong and T. Lorentzen: Acta Mater. 46 (1998) 3455-3466.
11) Y. Yamada and M. Taya: Mater. Trans., JIM 34 (1993) 254-260.

12) D. C. Lagoudas, J. G. Boyd and Z. Bo: Trans ASME, Eng. Mat. Tech. 116 (1994) 337-347.

13) M. Kawai, H. Ogawa, V. Baburaj and T. Koga: J. Intell. Mater. and Struct. 10 (1999) 14-28.

14) G. Murasawa, K. Tohgo and H. Ishii: J. Composite Mater. 38 (2004) 399-416.

15) D. C. Dunand, D. Mari, M. A. M. Bourke and J. A. Roberts: Metall. Mater. Trans. A 27A (1996) 2820-2836.

16) D. C. Lagoudas and I. G. Tadjbakhsh: Smart Mater. Struct. 1 (1992) 162-167.

17) J. G. Boyd and D. C. Lagoudas: J. Intell. Mater. Syst. and Struct. 5 (1994) 333-346.

18) G. Murasawa and K. Tohgo: Trans. Mater. Res. Soc. Japan 26 (2001) $163-166$.

19) G. Murasawa, S. Yoneyama, K. Tohgo and M. Takashi: Key Eng. Mater. 270-273 (2004) 2172-2178.

20) G. Murasawa, K. Tohgo and M. Takashi: Proc. International Conference on Advanced Technology in Experimental Mechanics 2003 (2003) CD-ROM publication.

21) E. Umezaki and T. Ichikawa: Int. J. Modern Physics B 17 (2003) 17501755 .

22) J. Ro and A. Baz: Composites Eng. 5 (1995) 61-75.

23) M. A. Sutton, S. R. Stephen McNeill, J. D. Helm and Y. J. Chao: Photomechanics (Springer-Verlag, 2000) pp. 323-372.

24) S. Yoneyama and Y. Morimoto: JSME International Journal Series A 46 (2003) 178-184.

25) D. R. Madill and D. Wang: IEEE Trans. Contr. Sys. Tech. 6 (1998) 473-481. 